\title{
Taxonomic and faunistic studies on the genus Harutaeographa (Lepidoptera, Noctuidae, Orthosiini) with description of a new species
}

\author{
Balázs Benedek ${ }^{1, \dagger}$, Aidas Saldaitis ${ }^{2, \ddagger}$, Jolanta Rimsaite ${ }^{3, \S}$ \\ I H-2045 Törökbálint, Árpád u. 53, Hungary 2 Nature Research Centre, Akademijos str. 2, LT-08412 Vil- \\ nius-21, Lithuania 3 Nature Research Centre, Akademijos str. 2, LT-08412 Vilnius-21, Lithuania \\ † urn:lsid:zoobank.org:author:39550B03-2A4C-4BA1-877D-00F15999FD33 \\ ¥ urn:lsid:zoobank.org:author:1C168900-4B0F-4999-895D-FAB0B16B14C1 \\ § urn:lsid:zoobank.org:author:9754C97B-5324-42CE-96F5-64E91E13C7E7 \\ Corresponding author: Jolanta Rimsaite (jolanta@ekoi.lt)
}

Academic editor: D. Lafontaine | Received 24 August 2012 | Accepted 26 October 2012 | Published 15 November 2012

urn:lsid:zoobank.org:pub:8A592F63-32BD-4929-96D2-484B4DFAOBBE

Citation: Benedek B, Saldaitis A, Rimsaite J (2012) Taxonomic and faunistic studies on the genus Harutaeographa (Lepidoptera, Noctuidae, Orthosiini) with description of a new species. ZooKeys 242: 51-67. doi: 10.3897/ zookeys.242.3889

\begin{abstract}
Description of a new Harutaeographa Yoshimoto, 1993 species, Harutaeographa shui sp. n. from China's Sichuan province, is given. Harutaeographa yangzisherpani transformis Hreblay \& Ronkay, 1999 is combined as a synonym of Harutaeographa yangzisherpani yangzisherpani Hreblay \& Ronkay, 1999. Additional distributional data for Harutaeographa pallida Yoshimoto, 1993, and Harutaeographa cinerea Hreblay \& Ronkay, 1998 are provided. A checklist of the genus Harutaeographa and a key to the Harutaeographa fasciculata (Hampson, 1894) species-group, based on external characters and genitalia, are presented.
\end{abstract}

\section{Keywords}

Orthosiini, new species, Harutaeographa, distribution

Copyright Balázs Benedek et al. This is an open access article distributed under the terms of the Creative Commons Attribution License 3.0 (CC-BY), which permits unrestricted use, distribution, and reproduction in any medium, provided the original author and source are credited. 


\section{Introduction}

This paper contributes additional taxonomic, genitalic and faunistic information on the taxonomy of the genus Harutaeographa Yoshimoto, 1993 to what was previously provided in Chen (1999), Hacker and Ronkay (1996), Hampson (1894, 1906), Hreblay and Ronkay (1998, 1999), Hreblay et al. (1998), Kononenko et al. (1998), Ronkay and Ronkay (2000), Ronkay et al. (2001, 2010), and Yoshimoto (1993, 1994). The genus Harutaeographa is typical of the tribe Orthosiini within the Himalayan Noctuidae and contains 37 species and 3 subspecies distributed mostly in the Southeast Asian and Himalayan regions. Except for a few Western-Himalayan and Central-Asian species inhabiting semidry areas, most members of this genus are associated with the Himalayan monsoonic forest belt. Flight periods generally extend through March and April, but some SouthernHimalayan species are on the wing during the colder November to February period.

\section{Materials and methods}

The specimens of Harutaeographa preserved in the collections of Alessandro Floriani (Milan, Italy), Balázs Benedek (Törökbálint, Hungary), Gottfried Behounek (Grafing, Germany)/Zoologische Staatssammlung, Munich (Germany), Danny Nilsson (Kalvehave, Denmark) and Nature Research Centre (Vilnius, Lithuania) were examined. The specimens examined were collected in China and Nepal using ultraviolet light traps and occasionally sugar ropes. Seventeen genital slides were prepared and 27 photographs were made. Examination of morphology: after maceration, male and female genitalia were dissected and mounted in euparal on glass sides. Dissection of genitalia follows Lafontaine (2004). Photographs of genitalia were made using a Wild M3Z microscope and Canon EOS 350D camera.

Abbreviations of the material depositories:

AFM Alessandro Floriani (Milan, Italy);

BBT Balázs Benedek (Törökbálint, Hungary);

BMNH Natural History Museum, London (United Kingdom);

DNK Danny Nilsson (Kalvehave, Denmark);

HNHM Hungarian Natural History Museum, Budapest (Hungary);

MNHU Museum für Naturkunde Leibniz Institute for Research on Evolution and Biodiversity, Berlin (Germany);

NHM Naturhistorisches Museum Wien (Austria);

NRCV Nature Research Centre (Vilnius, Lithuania);

NSMT National Science Museum Tokyo (Japan);

ZFMK Zoological Forschungsinstitut und Museum Alexander Koenig, Bonn (Germany);

ZMH Zoological Museum of Helsinki (Finland);

ZSM Zoologische Staatssammlung, Munich (Germany). 


\section{Systematic accounts}

Key to Harutaeographa species related to Harutaeographa fasciculata based on external characters

$1 \quad$ Forewings dark brown with black pattern or patches of dark scales.............2 Forewings light brown, orbicular stigma whitish (Figs 10,11)

H. fasciculata (Himalaya: Sikkim, N.India; Nepal)

2 Wingspan of forewings 37-42 mm, forewings narrow, cilia golden yellow (Figs 6, 7) .......................................................... shui (China: Sichuan) Wingspan of forewings $42-48 \mathrm{~mm}$, forewings wide, cilia dark brown (Figs 8 , 9) H. odavissa (China: Shaanxi, Hubei, Sichuan)

Key to Harutaeographa species related to Harutaeographa fasciculata based on genital characters

1 Valva at apex widening, clasper curved, ductus bursae straight

- Valva not widening at apex, clasper almost straight, ductus bursae curved at base (Figs 17, 23). H. fasciculata

2 Uncus short, apical part of cucullus a boot-shaped plate with short blunt apex, ampula long, curved at apex, almost perpendicular to costa, both anterior and posterior parts of corpus bursae almost equal in size (Figs 15, 21)..

H. shui sp. $\mathrm{n}$.

- Uncus $1 / 3$ longer than in $H$. shui, apical part of cucullus boot-shaped with short acute apex, ampula slightly curved at apex, posterior part of corpus bursae longer and narrower than anterior part (Figs 16, 22)....... H. odavissa

\section{Harutaeographa shui Benedek \& Saldaitis, sp. n.}

urn:Isid:zoobank.org:act:C3F72129-2643-4234-84F6-ABEA933B13A8

http://species-id.net/wiki/Harutaeographa_shui

Figs 6, 7, 15, 21

Type material. Holotype: male (Fig. 6), China, Sichuan, $29^{\circ} 43.105^{\prime} \mathrm{N}, 02^{\circ} 36.195^{\prime} \mathrm{E}$, near Siping, 1600 m, 27.iii.2011, Floriani leg., in the collection of ZSM; (slide No. JB1792m).

Paratypes: 3 males, with the same data as the holotype, 1 male, from the same locality, but 02.iv.2011, 1 female (Fig. 7), China, W. Sichuan, road Menghugang/ Kangding, $29^{\circ} 49.955^{\prime} \mathrm{N}, 102^{\circ} 02.827^{\prime} \mathrm{E}, 1500 \mathrm{~m}, 19$. iv.2010, leg. Chen Gun, in the AFM, BBT, and ZSM collections. Slide No. JB1793f.

Etymology. The specific name refers to the Shu Kingdom, which is now Chengdu, the capital of China's Sichuan province.

Diagnosis. Harutaeographa shui in general appearance is similar to $H$. fasciculata (Hampson, 1894) (Figs 10, 11), but is smaller and has more oblong forewings. Despite 
this superficial resemblance to $H$. fasciculata, based on genital morphology the new species is more closely related to Harutaeographa odavissa Ronkay, Ronkay, Gyulai \& Hacker, 2010 (Figs 8, 9). These species are easily distinguishable externally by forewing shape, coloration and pattern. The male genitalia differ from those of $H$. odavissa (Fig. 16) by its shorter uncus, smaller tegumen, remarkably broader and apically more elongate cucullus, and the configuration of the clasper-ampulla complex. The structure of aedeagus and vesica are similar to those of $H$. odavissa (Fig. 16), but H. shui (Fig. 15) has a slightly more curved aedeagus, differently configured vesica, and longer subterminal cornuti field. The female genitalia of the new species differ from those of $H$. odavissa (Fig. 22) in having a shorter ovipositor, shorter apophyses, and shorter and weaker ductus bursae.

Description. Wingspan 37-42 mm, length of forewing 17-20 mm. Head, front and thorax chocolate brown with some copper shine; male antenna bipectinate, female antenna narrow ciliate; forewings richly decorated with dark coppery-brown patterns distinctly marked with black scales, outer margin and cilia lighter golden yellow; hindwings with intensive dark suffusion, especially wide on outer margin, discal spot, and well-marked postmedial fascia; cilia with copper shine. Male genitaila (Fig. 15): uncus short, evenly broad; tegumen small and low; vinculum strong, narrow, V-shaped; valva finely arcuate; cucullus broad with apex elongate; sacculus weak, less sclerotized; clasper and ampulla robust outside and turned at middle; aedeagus rather long, gently arcuate; vesica with two subbasal coils and a small rasp-plate at base near carina, two small bunches of stronger cornuti in subbasal coils, and a long brush-like cornuti field on arcuate subterminal area. Female genitalia (Fig. 21): ostium nearly evenly truncated; ductus bursae narrow, somewhat wider and less sclerotized posteriorly; appendix bursae relatively small and rounded, weakly sclerotized; corpus bursae elongated, mesially constricted, with anterior and posterior parts subequal.

Bionomics and distribution. The new species is known only from Siping and Kangding areas of Sichuan Province (China), on the eastern edge of Tibetan plateau, where a few specimens were collected at the end of March - beginning of April at altitudes ranging from 1500 to $1600 \mathrm{~m}$. It was attracted to light during cold $\left(2-4{ }^{\circ} \mathrm{C}\right)$ nights in small river valleys. The habitat is mountain virgin mixed forest dominated by various broad-leaved trees, rhododendrons and bamboos (Fig. 27).

\section{Harutaeographa monimalis (Draudt, 1950)}

http://species-id.net/wiki/Harutaeographa_monimalis

Figs 4, 5, 18, 24

Material examined. 1 male, 1 female, China, W. Yunnan, Baiyunshan, 2600 m, Yunlong county, end of ii - early iii.2008, leg. Yi et al, in the collection of DNK; slide Nos JB1851m (Fig. 18); JB1852f.

Diagnosis. Male genitalia. Uncus small, elongated; tegumen short; juxta long, tongue-shaped, more or less quadrangular; vinculum short and heavily sclerotised, 


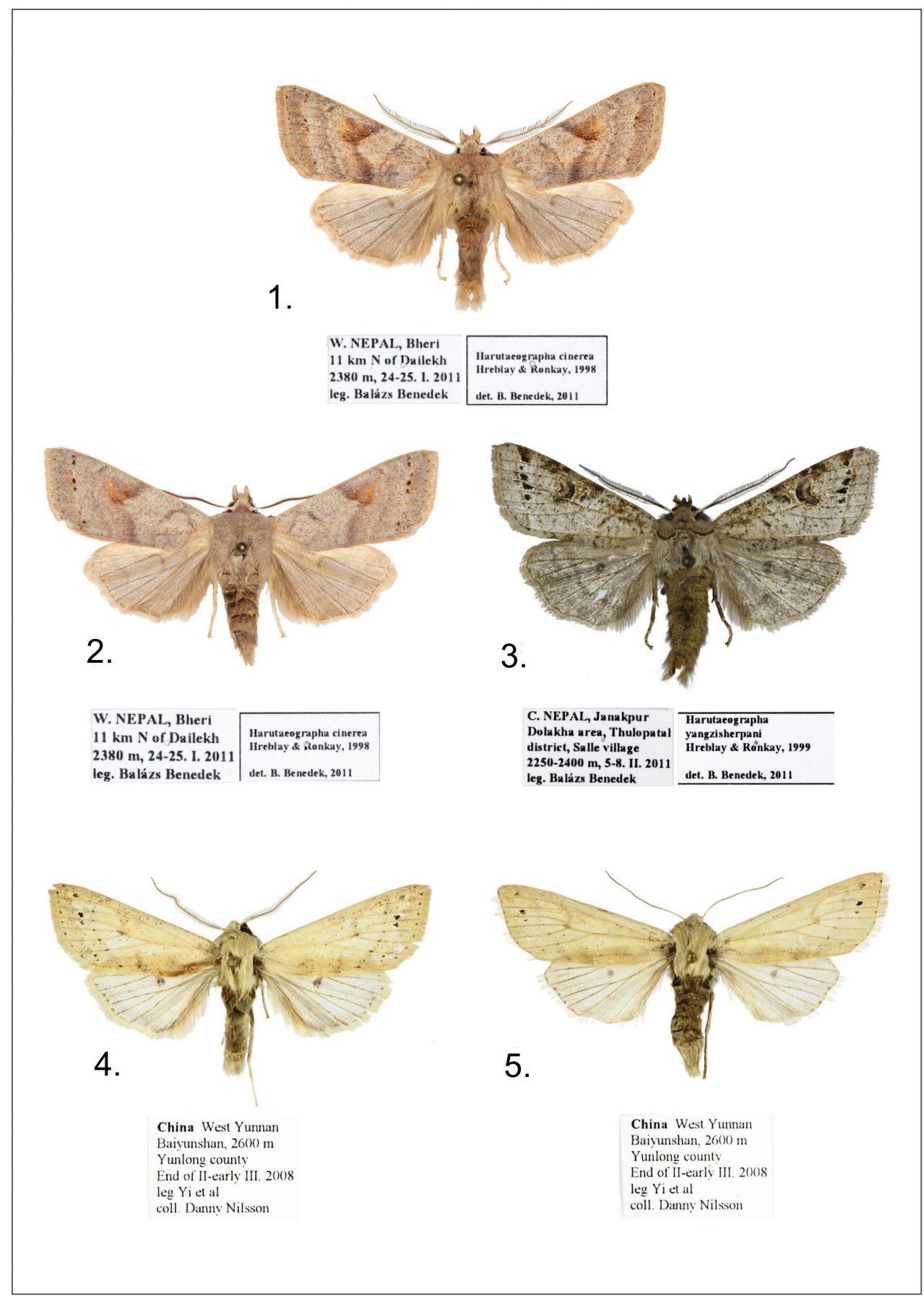

Figures I-5. Harutaeographa ssp. adults. I H. cinerea, male, West-Nepal, Bheri (BBT) 2 H. cinerea, female, West-Nepal, Bheri (BBT) 3 H. yangzisherpani yangzisherpani, male, Nepal, Janakpur (BBT) $4 H$. monimalis, male, China, W. Yunnan (DNK) 5 H. monimalis, female, China, W. Yunnan (DNK) 


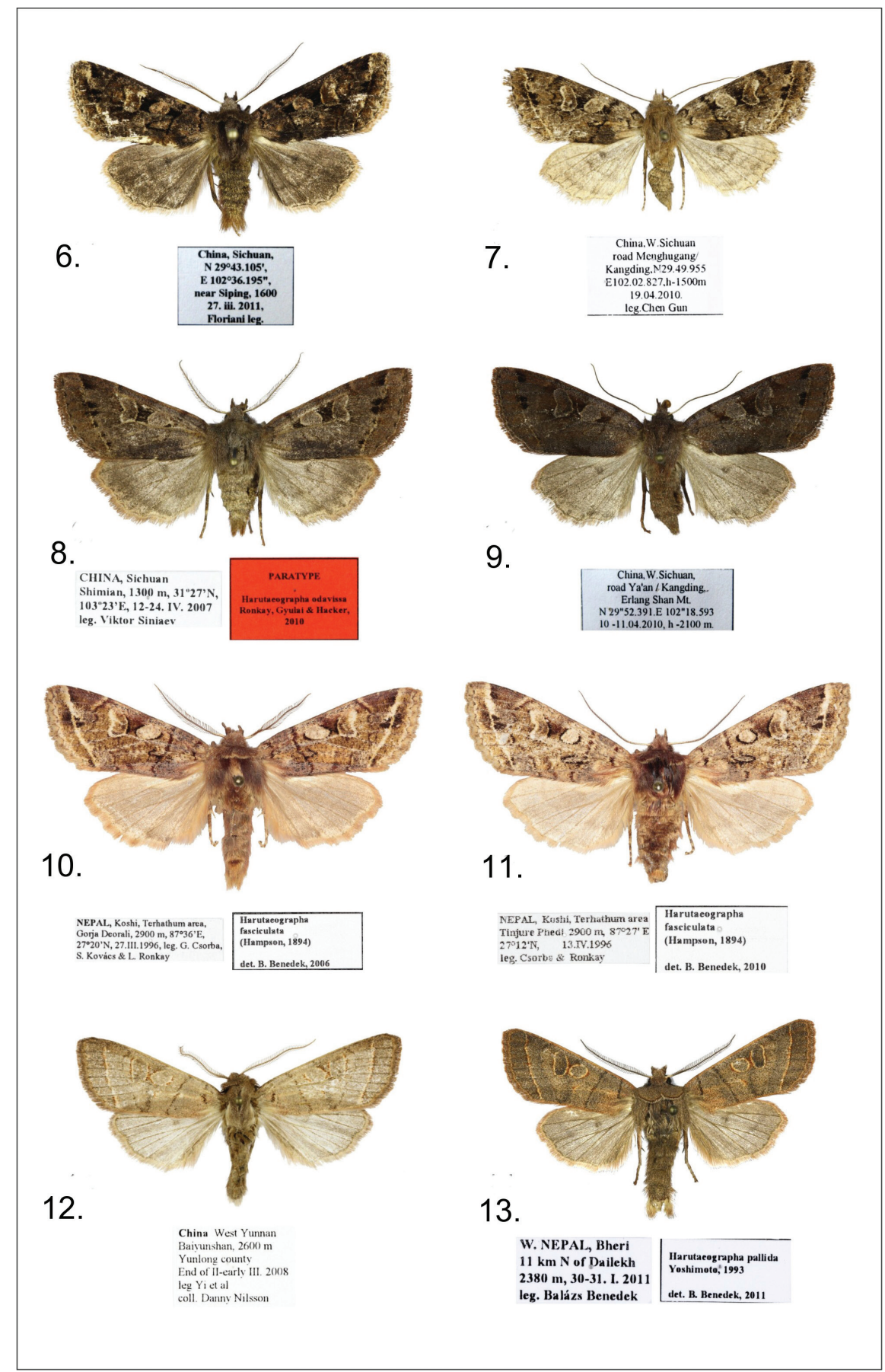

Figures 6-13. Harutaeographa ssp. adults. 6 H. shui, male, holotype, China, Sichuan (GBG/ZSM) 7 H. shui, female, paratype, China, Sichuan (AFM) 8 H. odavissa, male, paratype, China, Sichuan (BBT) 9 H. odavissa, female, China, Sichuan (BBT) I0 H. fasciculata, male, Nepal, Koshi (BBT) II H. fasciculata, female, Nepal, Koshi (BBT) $\mathbf{2}$ H. pallida, male, China, W. Yunnan (DNK) $\mathbf{3}$ H. pallida, male, West-Nepal, Bheri (BBT) 
U-shaped; sacculus broad, with more or less parallel margins. Clasper relatively large, thumb-like, with elongated and heavily sclerotised base; clasper fused with relatively small and evenly curved ampulla. Valvae more or less symmetrical, broad, armed with strong, finger-shaped ventral process and large, broad digitus; cucullus broad and strong, more or less rhomboidal in shape. Aedeagus relatively long, straight and broad; vesica evenly helicoid in shape, everted ventrally, covered with a row of fine spiculi from basal part of vesica along to terminal segment where it merges into a stouter cluster of longer spines forming a brush-like structure. Female genitalia (Fig. 24): Ostium bursae wide, rounded; ductus relatively short; appendix bursae helicoid with three coils; corpus bursae constricted mesially with both anterior and posterior sections broadly elliptical.

Note. This is the first new report of this species known previously only by its holotype specimen collected by Dr. H. Höne in 1935 (coll. ZFMK, Bonn).

\section{Harutaeographa yangzisherpani transformis Hreblay \& Ronkay, 1999, syn. nov.} http://species-id.net/wiki/Harutaeographa_yangzisherpani_transformis Figs 3, 19

Material examined. 2 males, Nepal, Janakpur, Dolakha area, Thulopatal district, Salle village, $2250-2400 \mathrm{~m}, 27^{\circ} 35.998^{\prime} \mathrm{N}, 86^{\circ} 09.775^{\prime} \mathrm{E} 5-8 . i i .2011$, leg. Balázs Benedek, in the collection of BBT; slide No. JB1807m (Fig. 19).

Note. The genitalia of H. yangzisherpani yangzisherpani Hreblay \& Ronkay, 1999 from Nepal exactly match those of Harutaeographa yangzisherpani transformis Hreblay $\&$ Ronkay from N. Vietnam pictured in Esperiana, 1999. The very slight genital differences between $H$. yangzisherpani yangzisherpani Hreblay \& Ronkay, 1999 and $H$. yangzisherpani transformis Hreblay \& Ronkay, 1999, suggest that they representing the same taxon and it is not reasonable to separate them as subspecies. Thus, H. yangzisherpani transformis Hreblay \& Ronkay, 1999 is combined here as a synonym of H. yangzisherpani yangzisherpani Hreblay \& Ronkay, 1999. It is first report of this species from Nepal.

\section{Harutaeographa pallida Yoshimoto, 1993}

http://species-id.net/wiki/Harutaeographa_pallida

Figs 12, 13, 20, 25

Material examined. 1 female, West-Nepal, Bheri, Dailekh area, $11 \mathrm{~km} \mathrm{~N}$ of Dailekh, 2380 m, 24-25.i.2011, 1 male, West-Nepal, Bheri, Dailekh area, $11 \mathrm{~km} \mathrm{~N}$ of Dailekh, 2380 m, 30-31.i.2011, 2 males, Nepal, Janakpur, Dolakha area, Thulopatal district, Salle village, 2250-2400 m, 5-8.i.2011, leg. Balázs Benedek, 3 males, 1 female, China, W. Yunnan, Baiyunshan, $2600 \mathrm{~m}$, Yunlong county, end of ii - early iii.2008, leg. Yi et $\mathrm{al}$, in the collections of BBT and DNK; slide Nos JB1849m, JB1850f, (Figs 20, 25).

Notes. These specimens, which are paler in colour than those from Nepal, are the first to be reported from China. 


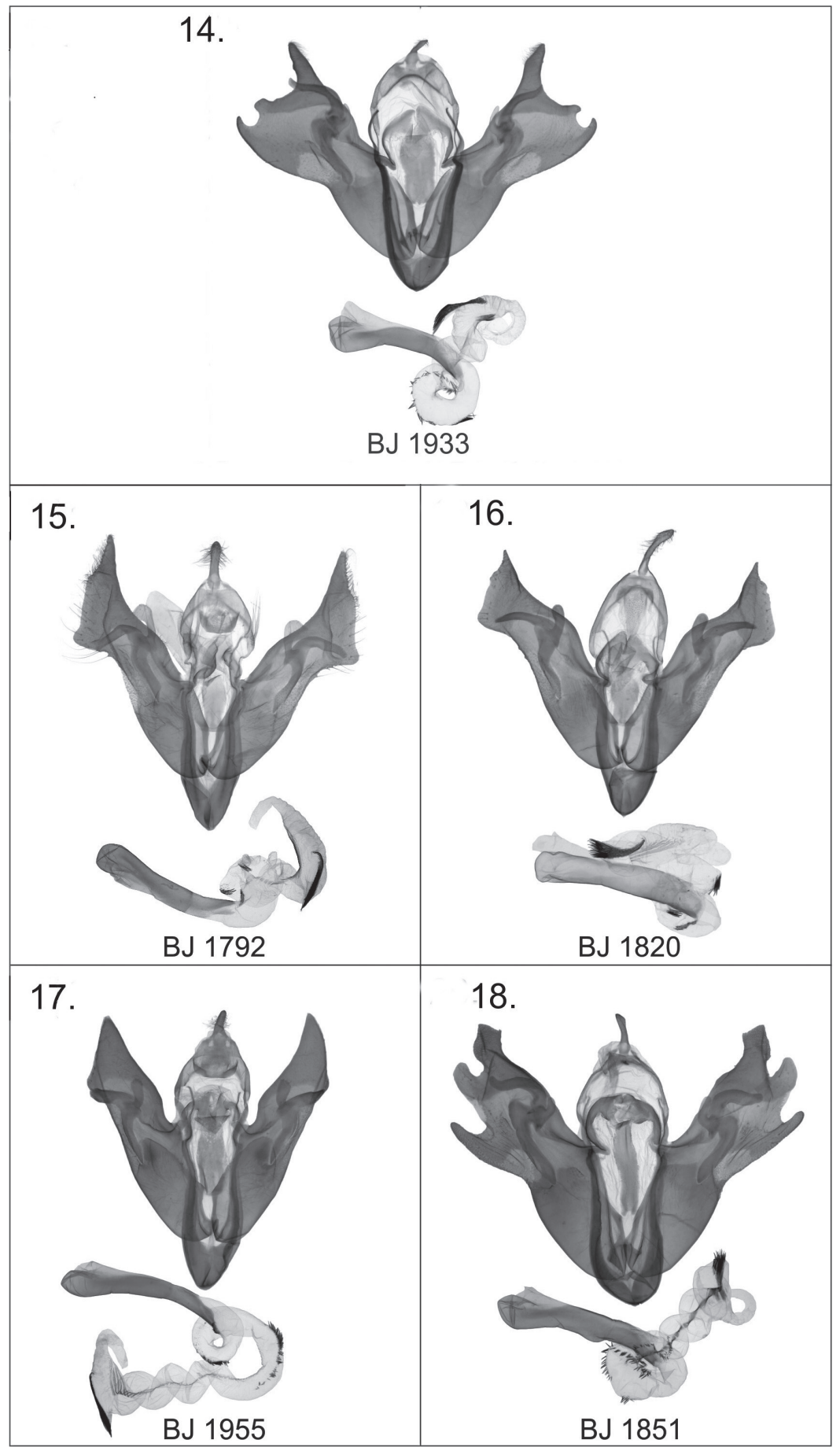

Figures I4-18. Harutaeographa ssp. male genitalia. I4 H. cinerea, male, prep. BJ1933m I5 H. shui, male, holotype, prep. BJ1792m I6 H. odavissa, male, paratype, prep. BJ1820m I7 H. fasciculata, male, prep. BJ1955m 18 H. monimalis, male, prep. BJ $1851 \mathrm{~m}$ 


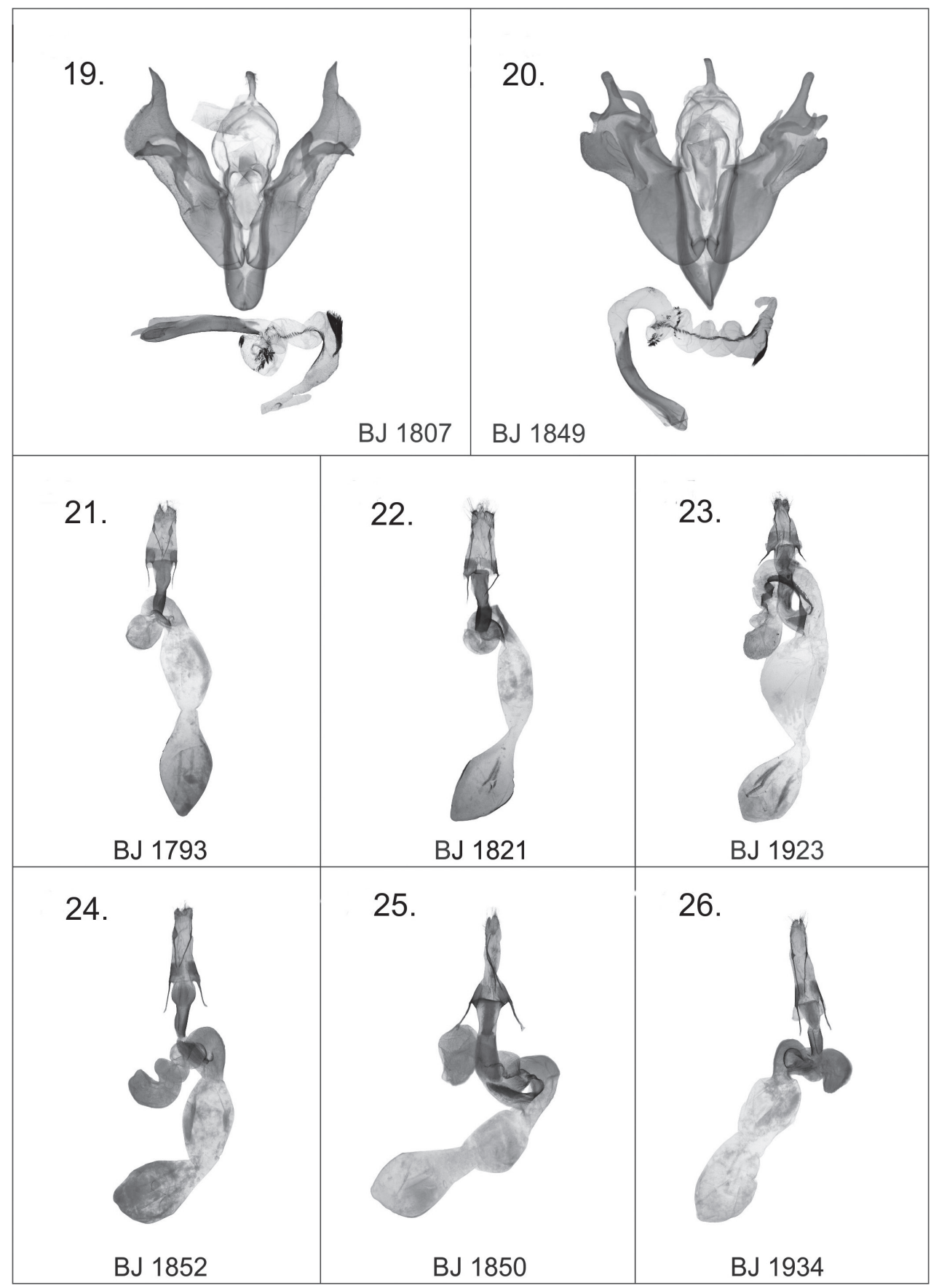

Figures 19-26. Harutaeographa ssp. male and female genitalia. 19 H. yangzisherpani yangzisherpani, male, prep. BJ1807m 20 H. pallida, male, prep. BJ1849m 2I H. shui, female, paratype, prep. BJ1793f 22 H. odavissa, female, prep. BJ1821f 23 H. fasciculata, female, prep. BJ1923f $\mathbf{2 4}$ H. monimalis, female, prep. BJ1852f 25 H. pallida, female, prep. BJ1850f 26 H. cinerea, female, prep. BJ1934f 


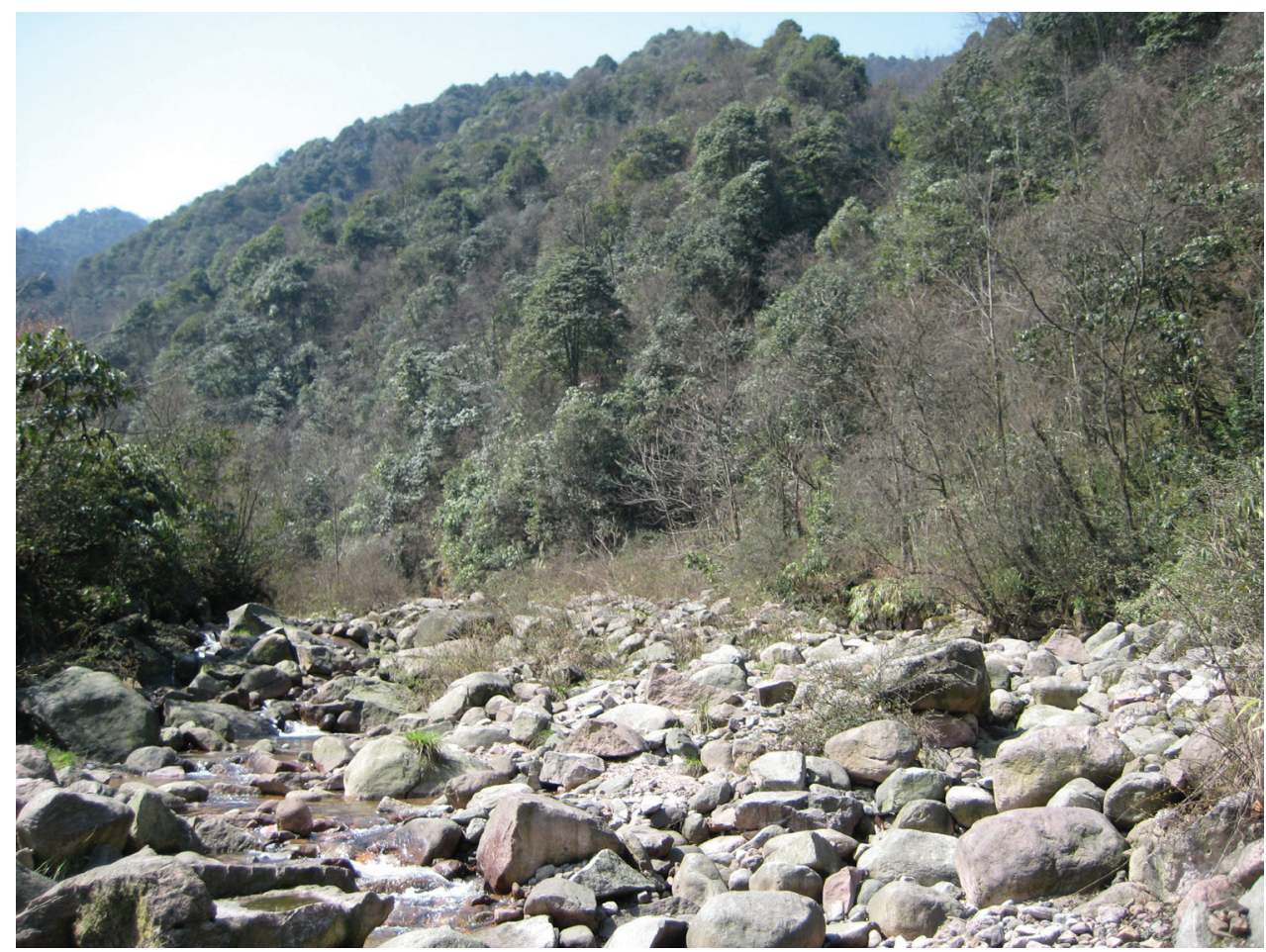

Figure 27. Harutaeographa shui, type locality, China, Sichuan near Siping.

\section{Harutaeographa cinerea Hreblay \& Ronkay, 1998}

http://species-id.net/wiki/Harutaeographa_cinerea

Figs 1, 2, 14, 26

Material examined. long series of both sexes from the following localities: WestNepal, Bheri, Dailekh area, $11 \mathrm{~km}$ N of Dailekh, 2380 m, 24-25.i.2011, West-Nepal, Bheri, Dailekh area, $13 \mathrm{~km} \mathrm{~N}$ of Dailekh, 2425 m, 26-27.i.2011, West-Nepal, Bheri, Dailekh area, $12 \mathrm{~km} \mathrm{~N}$ of Dailekh, $2600 \mathrm{~m}, 29 . i .2011$, West-Nepal, Bheri, Dailekh area, $11 \mathrm{~km} \mathrm{~N}$ of Dailekh, $2380 \mathrm{~m}, 30-31 . i .2011$, Nepal, Janakpur, Dolakha area, Thulopatal district, Salle village, 2250-2400 m, 5-8.ii.2011, leg. Balázs Benedek, in the collection of BBT.

Note. Little information has been available for this species due to insufficient collecting during its very early flight period (Hreblay and Ronkay 1998; Hreblay and Ronkay 1999). At the end of January-beginning of February in 2011 H. cinerea was one of the most frequently encountered noctuid species at light and sugar ropes at elevations between $2300-2600 \mathrm{~m}$ and is presumably widespread in Nepal. 


\section{Checklist of the genus Harutaeographa}

\section{Harutaeographa adusta Hreblay \& Ronkay, 1999}

Holotype: coll. M. Hreblay, HNHM. Type locality: Thailand, Changwat Chiang Mai, Mt. Doi Inthanon. Distribution: Indochina, Thailand.

\section{Harutaeographa akos Hreblay, 1996 [1997]}

Holotype: ZMH. Type locality: Tadjikistan, Gissar-Gebirge, Romit. Distribution: Tadjikistan (Gissar Mts.).

\section{Harutaeographa babai Sugi \& Sakurai, 1994}

Holotype: NSMT. Type locality: Nepal, Dhaulagiri, Jomsom. Distribution: Nepal; Himalaya.

\section{Harutaeographa bidui bidui Hreblay \& Plante, 1996 [1997]}

Holotype: coll. M. Hreblay, HNHM. Type locality: N.Pakistan, 5 km S Rattu. Distribution: Himalaya: N. Pakistan (Karakorum, Prov. Gilgit \& Baltistan, Rattu, Prov. Jammu \& Kashmir, Deosai Mts.).

\section{Harutaeographa bidui kaghanensis Hreblay \& Ronkay, 1999}

Holotype: coll. Hreblay, HNHM. Type locality: Pakistan, Prov. NW-Frontier, Kaghan valley, Khanian. Distribution: Pakistan (Prov. NW-Frontier, Kaghan valley, Khanian).

\section{Harutaeographa bicolorata Hreblay \& Ronkay, 1998}

Holotype: coll. Hreblay, HNHM. Type locality: Nepal, Ganesh Himal, 1 km E Gadrang. Distribution: Himalaya: Nepal (Ganesh Himal).

\section{Harutaeographa brahma Hreblay \& Ronkay, 1998}

Holotype: coll. Hreblay, HNHM. Type locality: Nepal, Ganesh Himal, 2 km W Thangjet. Distribution: Himalaya: Nepal (Ganesh Himal). 


\section{Harutaeographa brumosa Yoshimoto, 1994}

Holotype: NSMT. Type locality: Nepal, Janakpur, Jiri. Distribution: Himalaya: Nepal (Janakpur, Jiri, Terhathum distr.).

\section{Harutaeographa caerulea caerulea Yoshimoto, 1993}

Holotype: NSMT. Type locality: Nepal, Mt. Phulchouki. Distribution: Himalaya: Nepal (Katmandu Valley, Godavari).

Harutaeographa caerulea rubrigrapha Hreblay \& Ronkay, 1999

Holotype: coll. Hreblay, HNHM. Type locality: Thailand, Changwat Chang Mai, Mt. Doi Phahompok, 18 km NW Fang. Distribution: Indochina: Thailand (Chiang Mai).

\section{Harutaeographa castanea Yoshimoto, 1993}

Holotype: NSMT. Type locality: Nepal, Godavari. Distribution: Himalaya: Nepal (Kathmandu Valley, Godavari).

\section{Harutaeographa castaneipennis (Hampson, 1894)}

Holotype: BMNH. Type locality: India, Kashmir, Narkundah. Distribution: Himalaya: N.India (Prov. Jammu \& Kashmir).

\section{Harutaeographa cinerea Hreblay \& Ronkay, 1998}

Holotype: coll. G. Ronkay. Type locality: Nepal, Ganesh Himal, near Slya. Distribution: Nepal (Ganesh Himal, Slya).

\section{Harutaeographa craspedophora (Boursin, 1969)}

Holotype: coll. Vartian, NHM. Type locality: Afghanistan, Paghman-Gebirge, 20 km NW Kabul. Distribution: Afghanistan (Paghman Mts.).

\section{Harutaeographa diffusa Yoshimoto, 1994}

Holotype: NSMT. Type locality: Nepal, Janakpur, Jiri. Distribution: Himalaya: Pakistan; Nepal.

\section{Harutaeographa elphinia Hreblay \& Ronkay, 1999}

Holotype: HNHM. Type locality: Vietnam, Prov. Lao Cai, Mt. Fan-si-Pan, 7 km SW Sa Pa. Distribution: Indochina, Vietnam (Prov. Lao Cai, Tonkin). 


\section{Harutaeographa eriza (Swinhoe, 1901)}

Holotype: BMNH. Type locality: W. India, Punjab, Himachal Pradesh, Kulu. Distribution: Himalaya; Pakistan; India (Prov. Punjab).

\section{Harutaeographa fasciculata (Hampson, 1894)}

= H. fusciculata nec Hampson, 1894

Holotype: BMNH. Type locality: Sikkim (India). Distribution: Himalaya: Sikkim, N. India; Nepal; North Vietnam (Fansipan Mts).

\section{Harutaeographa ferrosticta (Hampson, 1894)}

Holotype: BMNH. Type locality: Kashmir, Narkundah. Distribution: Himalaya: Pakistan; N. India (Prov. Jammu \& Kashmir).

\section{Harutaeographa ganeshi Hreblay \& Ronkay, 1998}

Holotype: coll. G. Ronkay. Type locality: Nepal, Ganesh Himal, 2 km W Gholjong. Distribution: Himalaya: Nepal (Ganesh Himal, Gholjong).

\section{Harutaeographa izabella Hreblay \& Ronkay, 1998}

Holotype: coll. Hreblay, HNHM. Type locality: Nepal, Annapurna Himal, 1 km E Ghorepani. Distribution: Nepal.

\section{Harutaeographa kofka Hreblay, 1996 [1997]}

Holotype: BMNH. Type locality: N. India, Muktesar, Naini-Tal. Distribution: Himalaya: Pakistan; N. India; Nepal.

\section{Harutaeographa loeffleri Ronkay, Ronkay, Gyulai \& Hacker, 2010}

Holotype: coll. P. Gyulai, HNHM. Type locality: Burma, Chun state, Mindat camp. Distribution: Myanmar (Chun state, Mindat camp, Chun state, Natmataung Nationalpark, Mt. Victoria).

\section{Harutaeographa maria Hreblay \& Ronkay, 1999}

Holotype: coll. Hreblay, HNHM. Type locality: Pakistan, Prov. Jammu \& Kashmir, Naltar valley, $5 \mathrm{~km}$ E Naltar. Distribution: Himalaya: Pakistan (Prov Jammu \& Kashmir, Karakorum). 


\section{Harutaeographa marpha Hreblay \& Ronkay, 1999}

Holotype: coll. Hreblay, HNHM. Type locality: Nepal, Dhaulagiri Himal, 6 km NW Marpha. Distribution: Nepal; Himalaya.

\section{Harutaeographa monimalis (Draudt, 1950)}

Holotype: ZFMK. Type locality: China, Yunnan. Distribution: China (Prov. Yunnan).

\section{Harutaeographa odavissa Ronkay, Ronkay, Gyulai \& Hacker, 2010}

Holotype: HNHM. Type locality: China, Shaanxi, Taibaishan. Distribution: China (Prov. Shaanxi, Taibaishan, Tsinling Mts., Prov. Hubei, Daba Shan, Prov. Sichuan, Daxue Shan, Gongga Shan, Volong Reserve, Siguliang Shan, Qingcheng Shan).

\section{Harutaeographa orias orias Hreblay, 1996 [1997]}

Holotype: BMNH. Type locality: Prov. W. Bengal, Darjeeling. Distribution: Himalaya: N.India (Prov. Sikkim, Prov. W. Bengal, Darjeeling).

\section{Harutaeographa orias yoshimotoi Hacker \& Hreblay, 1996 [1997]}

Holotype: coll. Hacker, ZSM. Type locality: N. India, Himachal Prad., Rohtang. Distribution: Pakistan (Prov. Kashmir), Himalaya: N. India (Himachal Pradesh, Rohtang Pass, Prov. Sikkim); Nepal; Indochina; Thailand (Prov. Chiang Mai, Doi Phahompok)

\section{Harutaeographa pallida Yoshimoto, 1993}

Holotype: HNSMT, Tokyo (Japan). Type locality: Nepal, Godavari. Distribution: Himalaya: N. India (Prov. Sikkim); Nepal (Katmandu Valley, Godavari, Solu Khumbu Himal, Ganesh Himal); China (Prov. Yunnan).

\section{Harutaeographa pinkisherpani Hreblay \& Ronkay, 1998}

Holotype: coll. G. Ronkay. Type locality: Nepal, Ganesh Himal, 2 km SW Haku. Distribution: Himalaya: Nepal (Ganesh Himal, Haku).

Harutaeographa rama Hreblay \& Plante, 1996 [1997]

Holotype: coll. Hreblay, HNHM. Type locality: N.Pakistan, 10 km SW Astor, Rama. Distribution: Himalaya: Pakistan (Jammu \& Kashmir). 
Harutaeographa rubida (Hampson, 1894)

= H. bipuncta Yoshimoto, 1993

Holotype: BMNH. Type locality: Sikkim. Distribution: Himalaya: Nepal; N.India (Sikkim).

Harutaeographa saba Hreblay \& Plante, 1996 [1997]

Holotype: coll. M. Hreblay, HNHM. Type locality: N.Pakistan, 10 km SW Astor, Rama. Distribution: Pakistan; Afghanistan.

Harutaeographa seibaldi Ronkay, Ronkay, Gyulai \& Hacker, 2010

Holotype: coll. H. Seibald, Wien (Austria). Type locality: Burma, Chun state, Mindat camp. Distribution: Myanmar (Chun state, Mindat camp, Chun state, Natmataung Nationalpark, Mt. Victoria).

\section{Harutaeographa shui Benedek \& Saldaitis, 2012}

Holotype: ZSM. Type locality: China, Sichuan, $29^{\circ} 43.105^{\prime} \mathrm{N}, 102^{\circ} 36.195^{\prime} \mathrm{E}$, near Siping. Distribution: China (Sichuan).

Harutaeographa siva Hreblay, 1996 [1997]

Holotype: BMNH. Type locality: N. India, Simla. Distribution: Himalaya: N. India.

Harutaeographa stangelmaieri Ronkay, Ronkay, Gyulai \& Hacker, 2010

Holotype: coll. Becher/Stumpf (Germany). Type locality: China, Prov. Yunnan, Daxue Shan Mts. Distribution: China (Prov. Yunnan, Daxue Shan Mts).

\section{Harutaeographa stenoptera (Staudinger, 1892)}

Holotype: MNHU. Type locality: Ussuri, Amur. Distribution: Russia: (SE Siberia, Amur, Ussuri, Primorje); Korea; China (Shaanxi).

Harutaeographa yangzisherpani yangzisherpani Hreblay \& Ronkay, 1999

= H. yangzisherpani transformis Hreblay \& Ronkay, 1999

Holotype: coll. Hreblay, HNHM. Type locality: Thailand, Changwat Chiang Mai, Mts. Doi Inthanon. Distribution: Thailand, Vietnam; Nepal. 


\section{Acknowledgements}

The authors would like to express their sincere thanks to János Babics (Budapest, Hungary) for providing excellent genitalia preparations and photographs, Kulman Tamang (Kathmandu, Nepal) for field work assistance, Alessandro Floriani (Milan, Italy) and Danny Nilsson (Kalvehave, Denmark) for kindly loaning material, Gottfried Behounek (Grafing, Germany), Povilas Ivinskis (Vilnius, Lithuania) Rima Gulbinaite (Vilnius, Lithuania) for taxonomic suggestions, Tomas Zubacikas (Vilnius, Lithuania) for assistance with imago pictures and and especially to Mr Laszlo Ronkay (Hungary) and $\mathrm{Mr}$ Don Lafontaine (Canada) for corrections. The authors are especially grateful to Robert Borth (Milwaukee, United States) for English grammar suggestions.

\section{References}

Chen YCH (1999) Fauna Sinica: Insecta, Lepidoptera, Noctuidae, Vol. 16. Science Press, Beijing, 1596 pp.

Hacker H, Ronkay L (1996) Three new genera, seven new species and a general view of the late autumnal fauna of the Himachal Pradesh region in India (Lepidoptera, Noctuidae: Cuculliinae sensu Hampson). Esperiana, Buchreihe zur Entomologie 4: 337-359.

Hampson GF (1894) Fauna of British India (Moth) 2. Taylor and Francis, London, 609 pp. Hampson GF (1906) Catalogue of the Lepidoptera Phalaenae in the British Museum. Volume 6. British Museum Trustees, Taylor and Francis, London, 532 pp.

Hreblay M, Ronkay L (1998) Noctuidae from Nepal, 5. Tinea 15 (Suppl.1): 117-310.

Hreblay M, Ronkay L (1999) Neue trifide Noctuidae aus dem himalayischen Raum und der südostasiatischen Region, (Lepidoptera; Noctuidae). Esperiana, Buchreihe zur Entomologie 7: 485-620.

Hreblay M, Ronkay L, Plante J (1998) Contribution to the Noctuidae Fauna of Tibet and the adjacent regions (II.), a systematic survey of the Tibetan Noctuidae fauna based of the material of the Schäfer-Expedition (1938-1939) and recent expeditions (19381939). Part I, List of species collected by the Schäfer -Expedition. Part II, List of species of the expeditions 1996-1997. Part III, Descriptions. Esperiana, Buchreihe zur Entomologie 6: 69-184.

Kononenko VS, Ahn SB, Ronkay L (1998) Illustrated Catalogue of Noctuidae in Korea, (Lepidoptera), Insects of Korea. Series 3. Junghaeng-Sa, Seul, Korea, 507 pp.

Lafontaine JD (2004) Noctuoidea, Noctuidae (part), Noctuinae (part - Agrotini). In: Hodges RW (Ed) The Moths of North America, fasc. 27.1. The Wedge Entomological Research Foundation, Washington, 385 pp.

Ronkay G, Ronkay L (2000) New Agrochola Hübner, 1821 and Owadaglaea Hacker et Ronkay, 1996 species from Central Asia and Indochina (Lepidoptera, Noctuidae, Xylenini). Acta Zoolologica Academia Scientiarum Hungarica 46(4): 351-382. 
Ronkay G, Ronkay L, Gyulai P, Hacker H (2010) New Xylenini (Lepidoptera, Noctuidae, Xyleninae) species and genera from the wide sense Himalaya region. Esperiana, Buchreihe zur Entomologie 15: 245-354.

Ronkay L, Yela JL, Hreblay M (2001) Hadeninae II. Noctuidae Europaeae. Volume 5. Entomological Press, Sorø, 452 pp.

Yoshimoto H (1993) Moths of Nepal, Part 2, Noctuidae. Tinea 13 (Suppl.1): 124-141.

Yoshimoto H (1994) Moths of Nepal, Part 3, Noctuidae. Tinea 14 (Suppl.1): 95-139. 